\title{
SC CYCLOTRON AND RIB FACILITIES IN KOLKATA
}

\author{
Bikash Sinha, Rakesh Kumar Bhandari and Alok Chakrabarti (for the VECC Staff), Variable \\ Energy Cyclotron Centre, DAE, Kolkata 700 064, India
}

\section{Abstract}

Two advanced accelerator facilities are under construction at this centre, essentially, for basic nuclear physics experiments. Construction of the $\mathrm{K}_{\text {bend }}=520$ superconducting cyclotron for accelerating heavy ion beams is nearing completion. The main magnet was recently enrgized in the cyclotron vault. The excitation current is gradually being raised to reach the design value. Most of the major components/systems are being fabricated at various locations. We plan to start assembly of the cyclotron in the last quarter of 2005. Experimental facilities are also under design and construction by the user groups.

The Radioactive Ion Beam (RIB) facility will use the existing $\mathrm{K}=130$ room temperature cyclotron as the primary beam source and will be housed in a large experimental area. The major components of this facility consist of a target system, on-line ECRIS "charge breeder", a low beta RFQ and an IH linac. Several components of this complex facility have been developed or fabricated. The developemnt work on the others is going on. The design energy at the end of three linac tanks is about $400 \mathrm{keV} /$ nucleon, to be achieved around the end of 2006.

\section{INTRODUCTION}

The $\mathrm{K}=130$ cyclotron at the Centre has been delivering light heavy ion beams up to $\sim 15 \mathrm{MeV} /$ nucleon. Two ECR sources have been installed to produce the heavy ions one, primarily, for gaseous ion and the other for both gaseous as well as solid ions. The superconducting cyclotron under construction is expected to deliver up to $\sim 80 \mathrm{MeV} /$ nucleon with $\mathrm{K}_{\mathrm{foc}}=160$. The basic design features are similar to the cyclotrons operating at Michigan State University and Texas A\&M University in USA[1,2,3]. This jump in energy is expected to satisfy the experimental nuclear physics community as a whole.

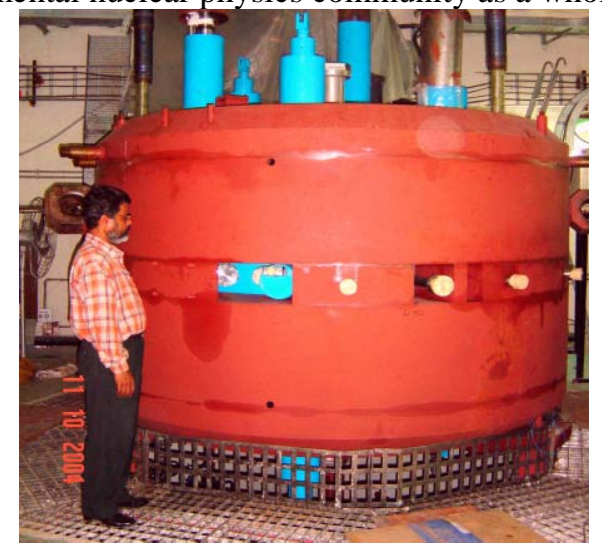

Figure 1: Cyclotron magnet with cryostat installed in it.
Energetic RIB projectiles have been the other requirement of this community. The RIB facility under development is $R \& D$ intensive. We have decided to build first all building blocks and to carry out, simultaneously, thick target $R \& D$ and release measurements using the existing ISOL facility utilizing the beams from $\mathrm{K}=130$ cyclotron. The design of the facility and some recent results are presented in this paper. Future expansion for further upgradation of energy has also been planned.

\section{STATUS OF THE SUPERCONDUCTING CYCLOTRON SYSTEMS}

\section{Main Magnet Frame}

The 80 tonne frame was fabricated in five major parts. The composition of the magnet iron has been made from same heat of steel to get near uniformity of chemical composition, physical homogeneity and grain size. The magnet frame assembly has been installed in the new cyclotron building (fig. 1).

\section{Superconducting Coil}

Winding of the main superconducting coil on the bobbin made from SS316L was completed in September 2003 as per specification. The coil winding was done in-house using an automatic winding set up developed based on our conceptual design by a reputed vendor. Strict quality control was enforced during coil winding. $\mathrm{Nb}-\mathrm{Ti}$ multifilamentary composite superconducting cable $\left(I_{c}=1030\right.$ A at 5.5 Tesla and $\left.4.2 \mathrm{~K}\right)$, consisting of 500 filaments of 40 micron diameter embeded in copper matrix was used. The parameters of the coils are given in the Table 1.

Table 1: Parameters of the coil

\begin{tabular}{|l|l|l|}
\hline \multirow{4}{*}{$\alpha$ coil } & No. of turns/coil & 1084 \\
\cline { 2 - 3 } & Total Length $(\mathrm{km})$ & 5.7 \\
\cline { 2 - 3 } & ID and OD $(\mathrm{mm})$ & 1521 and 1793 \\
\cline { 2 - 3 } & Inductance $(\mathrm{H})$ & 13.8 \\
\hline$\beta$ coil & No. of turns/coil & 2234 \\
\cline { 2 - 3 } & Length $(\mathrm{km})$ & 11.7 \\
\cline { 2 - 3 } & ID and OD $(\mathrm{mm})$ & 1521 and 1793 \\
\cline { 2 - 3 } & Inductance $(\mathrm{H})$ & 27.6 \\
\hline
\end{tabular}

\section{Cryostat}

The cryostat was assembled in the cyclotron building and placed on the magnet structure. Cryogenic instrumentation such as LHe level sensors, temperature sensors, strain gauges etc. have been installed inside the LHe chamber. This chamber has been insulated with multilayer insulation and LN2 cooled radiation shield assembled around it. The current leads, refrigeration lines and vent lines have also been welded to the LHe chamber 
and insulated. Finally, the whole insulated assembly has been welded inside the iron vacuum chamber (coil tank) as shown in the fig. 2. After the final assembly, helium leak test was performed.

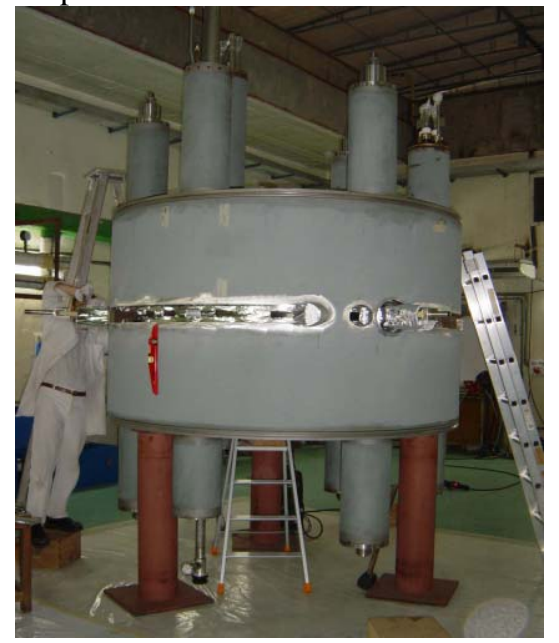

Figure 2: Coil tank without median plane inserts.

\section{Cryogen Delivery System}

The cryogen delivery system supplies cryogens, i.e., liquid helium and liquid nitrogen, to the main magnet cryostat and the cryopanels used for evacuating the acceleration chamber. The vacuum jacketed and liquid nitrogen shielded transfer lines have already been fabricated, tested and installed in the cyclotron building. At this time only the part of the ssystem that supplies cryogens to the cryostat has been installed. Various sensors for this cryogenic system, viz., temperature, level, pressure, strain, vacuum and their measuring units were tested thoroughly. The system is PLC controlled.

\section{Cooldown and Energization of the Main Coil[4]}

The main coil was cooled down to $4.5 \mathrm{~K}$ temperature after minimizing moisture contents in the liquid helium chamber to below $10 \mathrm{ppm}$ level. The cooldown time, starting from room tempereture, was about 3 weeks without cooling of the liquid nitrogen shield. This shield was, eventually, cooled after fixing air leaks in the current lead and vent port bellows. Subsequently, the coil was energized in a systematic manner, gradually, upto $550 \mathrm{~A}$ in both $\alpha$ and $\beta$ coils, at the time of writing this paper. Elaborate procedures to adjust the tension on radial supprt links were developed and followed during cooldown (fig. 3) and energization (fig. 4) of the coil. Details are described in another paper at this conference[4].

\section{Radiofrequency System}

The RF system has frequency range from 9 to $27 \mathrm{MHz}$ and the maximum attainable dee voltage about $100 \mathrm{kV}$. RF cavity components such as panels, movable shorts, contact fingers, drive mechanisms etc. have already been fabricated/purchased. Other major components such as the inner and outer conductors, dee shells, liners etc. are under fabrication. Major electronics components like power tubes, amplifiers, diagnostic equipment etc. are available at VECC. The PCBs for various circuits have been designed and getting fabricated.

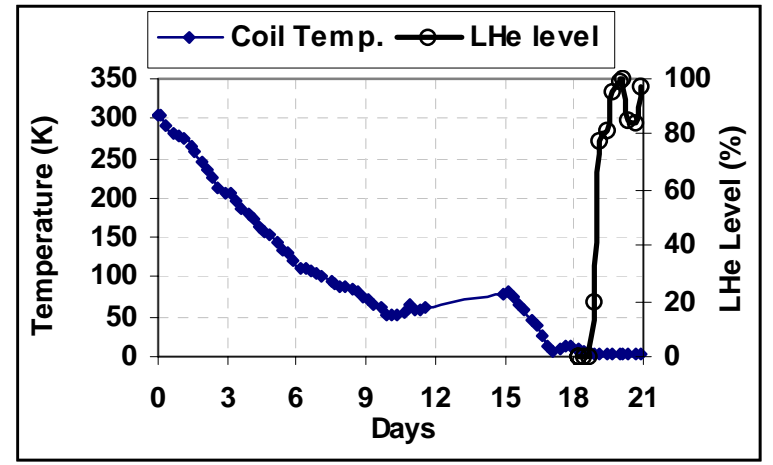

Figure 3: Coil temperature and LHe level without liquid nitrogen shield cooling.

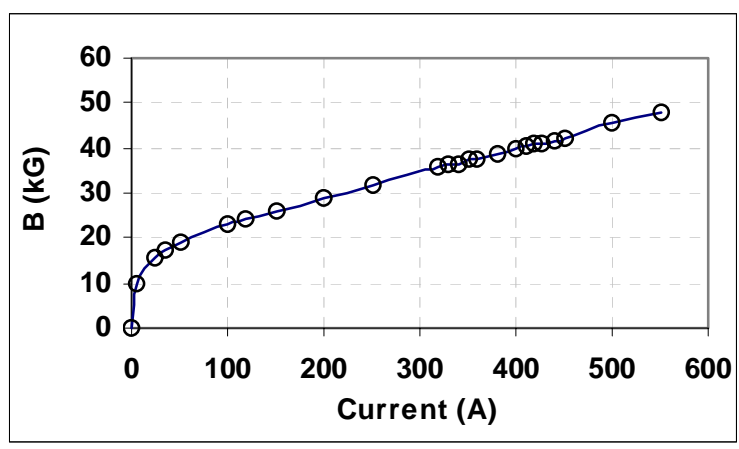

Figure 4: Magnetic field at 13" radius in hill region.

\section{Trim Coils}

All the 78 trim coils, made of water-cooled copper conductor, have been wound. Trials of vacuum impregnation with epoxy resin have been made. The trim coils will be mounted on the pole tips after first part of the field magnetic measurements and then vacuum impregnation will be done with epoxy resin.

\section{Magnetic Field Measurement System}

The magnetic field measurement set up has been installed in the magnet. Trial runs are being made to debug the systems. Extensive measurement of magnetic field and its harmonics has been planned to evaluate the errors and its correction. Accelerator physicists have developed codes for processing and analysing the measured field data. In order to correct for the field imperfections and to find out the operational parameters of the cyclotron for efficient beam acceleration and extraction, the orbit tracking codes, developed at MSU and VECC, will be used.

\section{Ion Source, Injection and Central Region}

In addition to the $14.5 \mathrm{GHz}$ ECR ion source already operating at VECC, an indigenously designed ECR ion source $(14.5 \mathrm{GHz})$ is being assembled. Elements of the injection beam line are under construction. In the beginning, the cyclotron will be operated in $1^{\text {st }}$ harmonic mode. The maximum possible dee voltage will be used to minimize the orbital path length. The accelerating 
electrode structure in the central region of the cyclotron has been designed. In fig. 5 three orbits are shown corresponding to starting time 240, 255 and 270 degrees, for $\mathrm{Z} / \mathrm{A}=0.357, \mathrm{~B}_{0}=35.04 \mathrm{kG}$, ion source extraction voltage $20 \mathrm{kV}$, extraction energy (E/A) $\sim 35 \mathrm{MeV} /$ nucleon and $\mathrm{V}_{\mathrm{dee}}=75 \mathrm{kV}$. Other ion species with various extraction energies and beam with finite emittance are being studied.

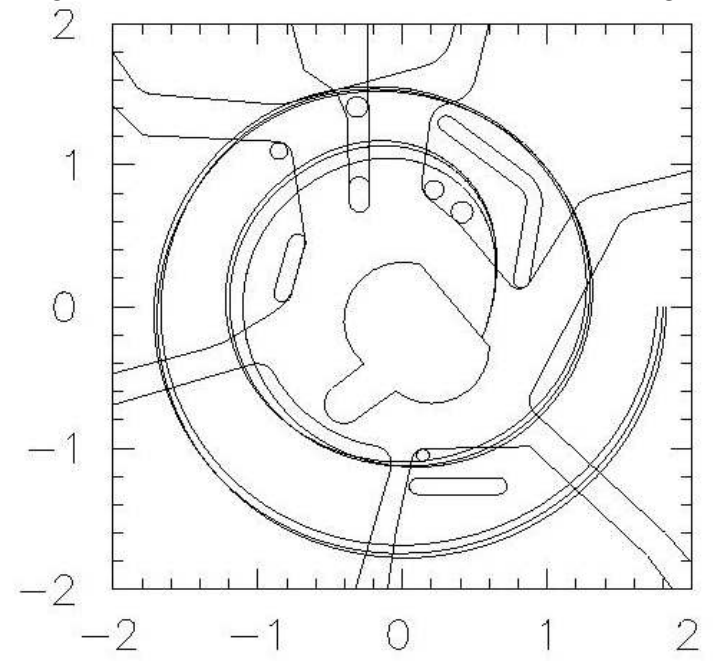

Figure 5: The central region electrode structure for $1^{\text {st }}$ harmonic mode of operation (scale in inches).

\section{Power Supplies}

The $10 \mathrm{ppm}$ class power supplies for the main coil along with air-cooled dump resistors has worked satisfactorily with actual load. Eighteen trim coil power supplies have been installed in the basement area. Prototypes of RF power supplies have been fabricated at the Centre. The most critical of these is the plate supply with 2 microseconds crowbar operation.

\section{Extraction System}

The extraction system consists of two electrostatic deflectors, eight passive magnetic channels, one active channel and compensating bars. Beam dynamics through the extraction system has been calculated. Minor modification may be done after field measurements. An active M9 channel is being developed.

\section{Control System and Beam Diagnostics}

The beam diagnostics inside the cyclotron will comprise of the main probe having three fingers and a differential probe in addition to a borescope for viewing the beam. Magnetic channels will have current readout to help in extraction optimisation. Capacitive as well as scintillation detector phase probes and image digitisation are being studied. A distributed control system connected through ETHERNET LAN and using mostly PCs with Windows and UNIX based systems will be used. Single and multi-tasking applications have been developed.

\section{Beamlines and Experimental Facilities}

Layout of the experimental areas is shown in figure 6. Calculations on the beam steering and phase space matching, immediately after extraction from the cyclotron, have been carried out. The external beam transport system uses two bending magnets to reduce the dispersion coefficients to a certain extent. The ion optics beyond the second bending magnet is telescopic in nature. Experimental facilities being developed include a large multipurpose scattering chamber, a $4 \pi$ charged particle array, high energy gamma detector array, irradiation facilities etc.

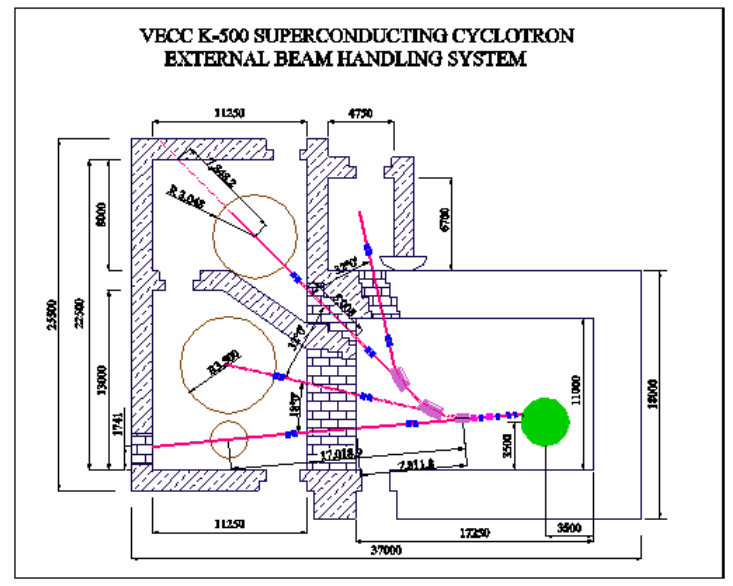

Figure 6: Beamlines for the superconducting cyclotron.

\section{RIB PROJECT}

A schematic layout of the facility outlining the plan up to the year 2007 is shown in fig. 7. Radioactive nuclei will be produced inside thick targets using proton and $\alpha$ particle beams from the $\mathrm{K}=130$ cyclotron at VEC. Multiply charged radioactive ions with $\mathrm{q}>1^{+}$will be produced in a charge breeder consisting of a surface ionsource coupled to a $6.4 \mathrm{GHz}$ on-line ECRIS. The desired RI Beam with an energy of $1 \mathrm{keV} /$ nucleon and $\mathrm{q} / \mathrm{A}=1 / 16$ will be separated in the low energy beam transport line after the ECRIS and accelerated to about $86 \mathrm{keV} /$ nucleon in a heavy-ion RFQ linac. Subsequently, the RI Beams will be accelerated to the desired final energy using heavy-ion IH linac. A brief description of the various systems is given below.

\section{Thick Target $R \& D$}

The production of radioactive ions is proportional to the thickness of the target, that is, on the number of target atoms per unit area of the target apart from primary beam intensity and nuclear reaction cross-section. In order to achieve fast and efficient release of activity from the target, the trick is to maximize surface area so that diffusion is enhanced. We have worked out in detail the prospect of using fibrous and composite targets like carbon fibre (RVCF) as backing matrix for deposition of several selected target compounds. Optimum target lengths for specific choices of RI beam with maximum allowable beam current are calculated for a number of targets.

As a first initiative in this direction, we have deposited pure 'Al' (17.6 mg) on carbon fibres (RVCF shown in 
fig. 8) to test the release of isotopes from the composite target, experimentally. The target was tested for release efficiency with $140 \mathrm{MeV}{ }^{16} \mathrm{O}$ beam. The release of ${ }^{38} \mathrm{~K}$ was monitored and close to $100 \%$ efficiency was observed [5].

\section{The "Two-Ion-Source" Charge Breeder}

In order to produce high charge states of RI ions with good enough efficiency the vacuum inside the on-line ECR ion-source needs to be kept $\sim 10^{-7}$ torr. This cannot be achieved if the ECRIS is kept in close vicinity of the thick target due to the residual gas pressure. Moreover, the permanent magnets of the ECRIS may undergo radiation damage in the hostile environment close to target. The possible solution is what is called a "two-ion source" charge breeder consisting of two ion-sources in tandem; a $1^{+}$thick-target integrated ion-source coupled to an $\mathrm{n}^{+}$ECRIS. This concept has been successfully tested at Grenoble for $\mathrm{n}^{+}$production of $\mathrm{Ar}, \mathrm{Rb}, \mathrm{Zn}, \mathrm{Pb}$ and other elements.

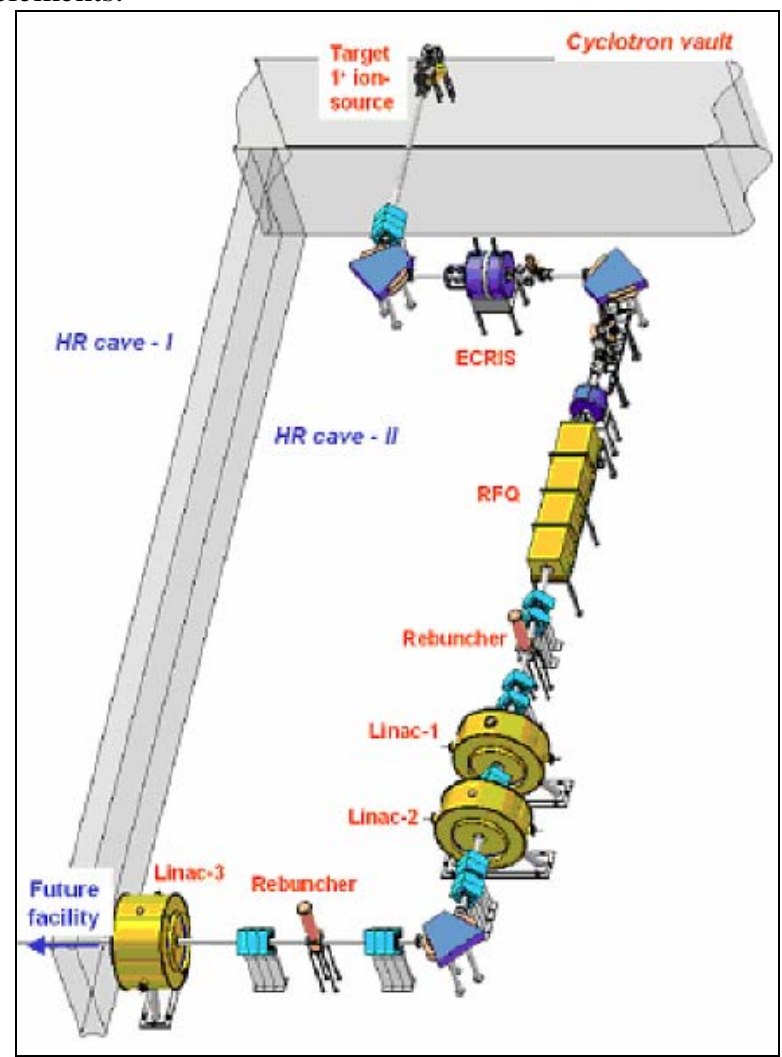

Figure 7: RIB Facility Layout.

The charge breeder for the VEC RIB[6] facility consists of a surface ionization source coupled to a $6.4 \mathrm{GHz}$ on-line ECRIS. The $1^{+}$ions from the first ionsource are decelerated to about $20-50 \mathrm{eV}$ and focused into the ECRIS plasma so that they can be efficiently trapped and further ionised to charge state $q>1^{+}$. A scheme for stepwise and gradual deceleration consisting of a multielectrode decelerator and a tuning electrode placed outside the ECRIS plasma chamber ensures soft landing of the $1^{+}$beam.
He-jet technique offers an alternative means of transferring short-lived radioactive atoms produced in the target chamber to the ECRIS. The first and second chambers shown in fig 9 represent the skimmer-1 and skimmer-2, respectively, which ensures proper vacuum level of about $1 \times 10^{-6}$ torr in the ECR plasma chamber. The small opening inside the chambers and pressure distribution decide the divergence of the activity at the ECR plasma position and also the transport efficiency.
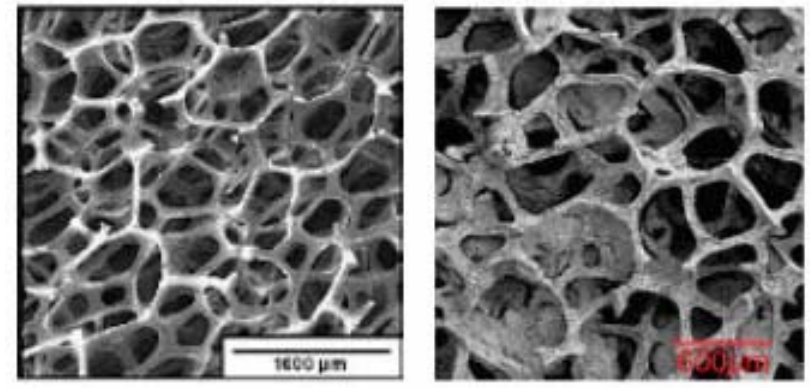

Figure 8(a) RVCF (b) RVCF coated with $\mathrm{Al}_{2} \mathrm{O}_{3}$ Powder.

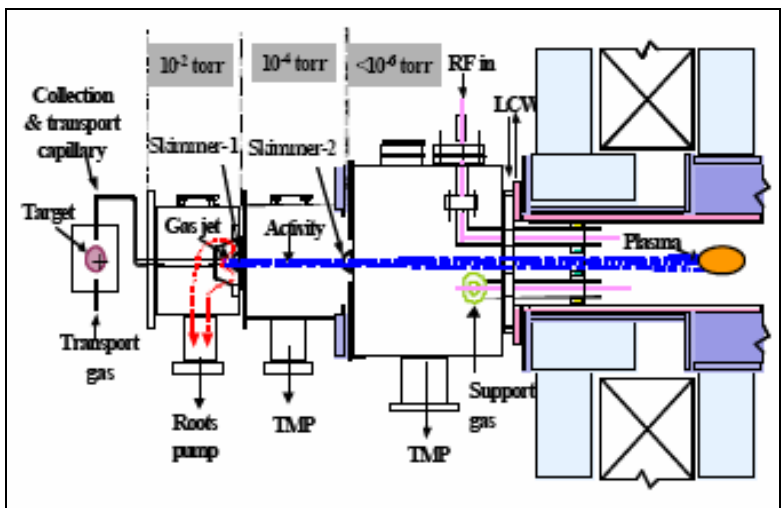

Figure 9: He Jet two stage skimmer.

The ECR-RFQ beam line transport the $1 \mathrm{keV} /$ nucleon beam extracted from ECR and matches with the acceptance of RFQ. The basic design consists of a separation stage followed by matching section. The separation stage is designed for dispersion of $1.94 \mathrm{~cm} / \%$.

\section{The Heavy Ion "RFQ” Post Accelerator}

For the VECC-RIB project a four-rod type RFQ linac has been designed [7] for an input beam energy of $1.0 \mathrm{keV} /$ nucleon and $q / A \geq 1 / 16$. The output energy will be $\approx 86 \mathrm{keV} /$ nucleon for a $3.2 \mathrm{~m}$ long, $35 \mathrm{MHz}$ structure. The $3 \mathrm{~d}$-co-ordinates of the vanes have been calculated using the program CUT3d. The process of surfacing, modelling as well as ball cutter profile and tool path generation for the vanes is done using CATIA. A typical vane profile is shown in figure 10. 


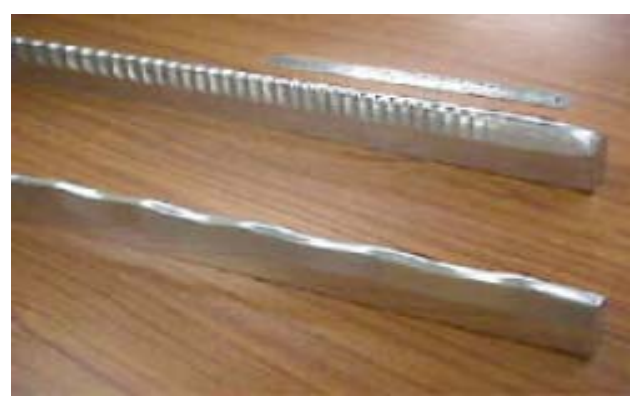

Figure 10: Vane profile of RFQ.

A half-scale cold model with un-modulated vanes has been fabricated to carry out RF structure studies. The Q \& Rp values obtained were both more than $50 \%$ of the MAFIA estimates. The resonance frequency was measured to be about $4 \%$ higher than the MAFIA estimate.

\section{The RFQ to LINAC Beamline}

An output beam of about $85.5 \mathrm{keV} /$ nucleon from the RFQ would be injected into the $35 \mathrm{MHz}$ IH-Linac (fig. 11) for acceleration to about $158 \mathrm{keV} /$ nucleon in the first tank. The RFQ to Linac beamline is designed to match the emittance of the beam at the exit of RFQ with the acceptance of the Linac. The design of the transverse optics is done by using two sets of quadrupole doublets. The longitudinal focusing is achieved with the help of a 4-gap re-buncher operating at $35 \mathrm{MHz}$.

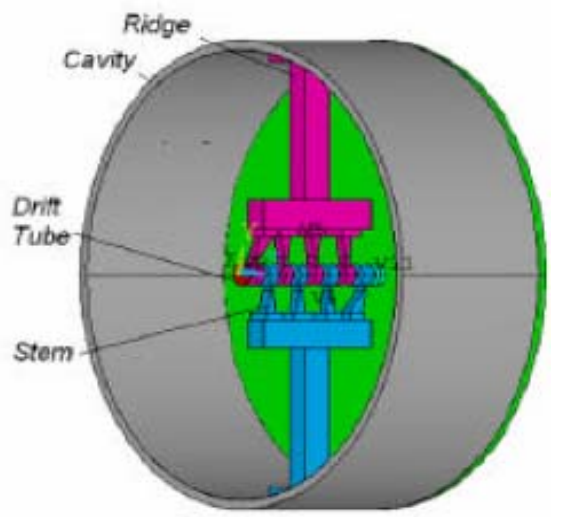

Figure 11: Schematic diagram of IH-LINAC tank.

The design of the first three LINAC tanks has been frozen and the fabrication of cold model for the Linac first tank has been completed. The cavity and the end covers were made out of aluminum. The drift tubes and ridges were also made out of aluminium. The measured and calculated frequency values match reasonably well whereas the measured Q-values are in the range of 35$45 \%$ of the calculated values. Two main causes of lower Q-values are surface imperfections of the inner components (drift tubes and ridges) and use of casting technique and imperfect joints in our cold model.

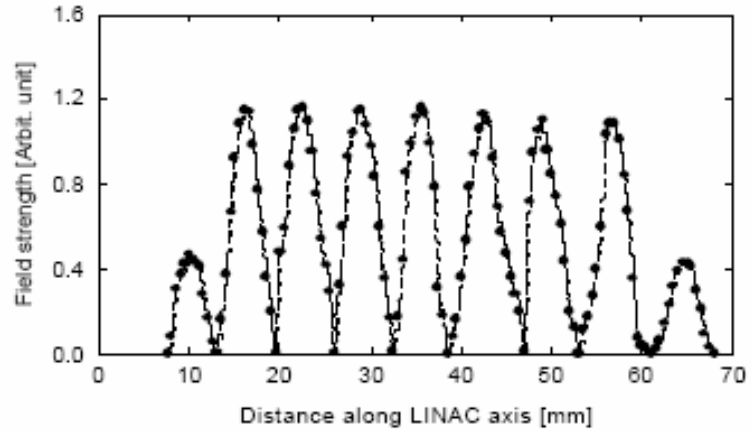

Figure 12: Axial field pattern in the cold model of the first linac cavity

The axial component of the RF electric field was measured using bead perturbation technique using an insulating teflon spherical ball. The arrangement allowed us to measure frequency deviation at a step of $5 \mathrm{~mm}$. Results of this measurement shows reasonably uniform field strength pattern in all the gaps shown in Figure 6.

\section{ACKNOWLEDGEMENTS}

Construction of the superconducting cyclotron and RIB facilities is dedicated teamwork of several scientists, engineers and administrative staff of VECC. We are grateful to all of them for their contribution.

\section{REFERENCES}

[1] H.G. Blosser, "The Michigan State University Superconducting Cyclotron Program," IEEE Trans. on Nuclear Science, Vol. NS-26, No.2, April 1979, p.2024.

[2] D.P. May et. al., "Status of the Texas A\&M University K500 Superconducting Cyclotron," Proc. $10^{\text {th }}$ Int. Conf. on Cyclotrons and their Applications, April 30-May 3, 1984, ed. F. Marti, p. 195.

[3] R.K. Bhandari, "Status of the Calcutta K500 Superconducting Cyclotron Project," $16^{\text {th }}$ Intl. Conf. on Cyclotrons and their Applications, May 13 - May 17 2001, edited by F. Marti, p. 84.

[4] Rakesh K. Bhandari and Bikash Sinha, "Commissioning of the Main Magnet of Kolkata K500 Superconducting Cyclotron," paper at this conference.

[5] D. Bhowmick et. al., "Preparation \& optimization of targets for the production of radioactive ions at VECC", Nucl. Instr. and Methods A539 (2005)54.

[6] V. Banerjee et. al., "Design of two ion source (2-IS) beam transport line for the production of multi charge radioactive ion beams", Nucl. Instr. and Methods A447 (2000)345.

[7] A. Chakrabarti et. al., "The design of four rod RFQ linac for the VEC-RIB facility", Nucl. Instr. and Methods A535 (2004)599. 